\title{
AN EXAMPLE CONCERNING CORE MEASURES
}

\section{S. J. SIDNEY ${ }^{1}$}

ABSTRACr. We shall construct a uniform algebra having a multiplicative linear functional whose space of representing measures is infinite-dimensional but nevertheless has a core measure. This answers in the negative an open question in Gamelin's book $[1, \mathrm{p} .106]$.

Let $\Delta=\{Z \in C:|Z| \leqq 1\}$, the closed unit disc, and let $T$ be its boundary. The disc algebra $A$ consists of those $f \in C(T)$ which have extensions $f \in C(\Delta)$ analytic on the interior of $\Delta$. We assume a basic familiarity with this algebra. In particular, if $Z \in \Delta$ and $\mu$ is a positive Borel measure on $T$ such that $\int f d \mu=\hat{f}(Z)$ for all $f \in A$, then either $\mu$ is point mass at $Z$ (if $Z \in T$ ) or $\mu=P \lambda$ where $\lambda$ is normalized Lebesgue measure on $T$ and $P$ is the Poisson kernel for $Z$ (if $Z \in \Delta / T$ ). This information, and the notions of representing measure and core measure, are in [1].

For $0 \leqq n \leqq \infty$ let $T_{n}=T \times\left\{p_{n}\right\} \subset C^{2}$ where $p_{0}=2, p_{\infty}=0$ and $p_{n}$ $=1 / n, 1 \leqq n<\infty$. Let $X=\bigcup\left\{T_{n}: 0 \leqq n \leqq \infty\right\}$, a compact set. Let $\eta_{n}: T \rightarrow X$ be $\eta_{n}(Z)=\left(Z, p_{n}\right)$. If $f \in C(X)$ let $f_{n}=f \circ \eta_{n} \in C(T)$. If $\mu$ is a finite Borel measure on $X$, let $\mu_{n}=\mu \circ \eta_{n}$, a finite Borel measure on $T$. Then $\sum_{0 \leq n \leq \infty}\left\|\mu_{n}\right\|=\|\mu\|$ and, for each $f \in C(X)$,

$$
\int f d \mu=\sum_{0 \leqq n \leqq \infty} \int f_{n} d \mu_{n} .
$$

This effects a one-to-one linear correspondence between measures on $X$ and sequences $\left\{\mu_{n}\right\}$ of measures on $T$ satisfying $\sum_{0 \leq n \leq \infty}\left\|\mu_{n}\right\|<\infty$. Note also that $0 \leqq \mu \leqq C \nu$ if and only if $0 \leqq \mu_{n} \leqq C \nu_{n}$ for all $n$, where $C$ is a nonnegative constant.

Fix a sequence (to be specified later) $\left\{Z_{n}\right\}_{1 \leq n<\infty}$ of distinct complex numbers with $0<\left|Z_{n}\right|<1$ and $Z_{n} \rightarrow 1$, and let

$\mathfrak{A}=\left\{f \in C(X): f_{n} \in A, 0 \leqq n \leqq \infty\right.$, and $\left.\hat{f}_{n}\left(Z_{n}\right)=\hat{f}_{0}\left(Z_{n}\right), 1 \leqq n<\infty\right\}$.

Clearly $\mathfrak{A}$ is a uniformly closed subalgebra of $C(X)$ containing the constants. The functions we will construct below show that $\mathfrak{A}$

Received by the editors February 16, 1970.

A MS 1969 subject classifications. Primary 4655.

Key words and phrases. Core measure, uniform algebra, multiplicative linear functional, Poisson kernel.

1 Research supported in part by National Science Foundation grant GP-16392. 
separates points on $X$ except for the pair $\{(1,0),(1,2)\}$, so is a uniform algebra on the quotient space $Y$ obtained from $X$ by identifying this pair to a point. Let $\phi$ be the multiplicative linear functional $\phi(f)=\hat{f}_{0}(0)$ on $\mathfrak{A}$, and let $M_{\phi}$ denote the (convex, weak* compact) set of positive Borel measures $\mu$ on $X$ representing $\phi$, i.e., $\int f d \mu=\phi(f)$ for all $f \in \mathfrak{A}$. Fix $\mu \in M_{\phi}$.

Suppose $F \in A$ and $\hat{F}\left(Z_{N}\right)=0$ for some $N, 1 \leqq N<\infty$. Define $f \in \mathfrak{A}$ by $f_{N}=F$ and $f_{n}=0, n \neq N$. Then by (1) $\int F d \mu_{N}=\int f d \mu=\phi(f)=0$. Thus there is a constant $C_{N} \geqq 0$ such that $\mu_{N}=C_{N} P_{N} \lambda$ where $P_{N}$ is the Poisson kernel for $Z_{N}$.

Next suppose $F \in A$ and $F(1)=0$. Define $f \in \mathfrak{A}$ by $f_{0}=0, f_{\infty}=F$ and $f_{n}=F-\hat{F}\left(Z_{n}\right), \quad 1 \leqq n<\infty$. By (1) $\int F d \mu_{\infty}=\int f d \mu=\phi(f)=0$, so there is a constant $C_{\infty} \geqq 0$ such that $\mu_{\infty}=C_{\infty} \delta$ where $\delta$ is point mass at 1.

Finally suppose $F \in A$ and define $f \in \mathfrak{A}$ by $f_{n}=F$ for all $\mathrm{n}$. By (1) $\hat{F}(0)=\phi(f)=\int f d \mu=\sum_{0 \leqq n \leqq \infty} \int F d \mu_{n}=\int F d\left(\sum_{0 \leq n \leq \infty} \mu_{n}\right)$, so $\sum_{0 \leq n \leq \infty} \mu_{n}=\lambda$. Thus $C_{\infty}=0$ and we see that the form of the sequence $\left\{\mu_{n}\right\}$ is

$$
\begin{aligned}
& \mu_{n}=C_{n} P_{n} \lambda, \quad 1 \leqq n<\infty, \\
& \mu_{0}=\left(1-\sum_{1 \leq n<\infty} C_{n} P_{n}\right) \lambda, \\
& \mu_{\infty}=0,
\end{aligned}
$$

where $\left\{C_{n}\right\}_{1 \leqq n<\infty}$ is a sequence of nonnegative numbers satisfying

$$
\sum_{1 \leqq n<\infty} C_{n} P_{n} \leqq 1
$$

Conversely, it is easy to see that any measure given by (2), (3) is in $M_{\phi}$. Note that $M_{\phi}$ consists of measures which assign no mass to points, hence may be identified with the space of representing measures for $\mathfrak{A}$ on $Y$.

Let $\gamma_{n}=\max P_{n} \geqq 1,1 \leqq n<\infty$. If for $1 \leqq N<\infty$ we define $\mu^{N}$ by (2) with $C_{N}=\gamma_{N}^{-1}$ and $C_{n}=0, n \neq N$, then the $\mu^{N}$ are affinely independent members of $M_{\phi}$, so the latter is infinite dimensional.

It remains to select the $Z_{n}$ so that $M_{\phi}$ has a core measure. Fix a sequence $\left\{\theta_{n}\right\}_{1 \leqq n<\infty}$ of distinct nonzero numbers in $(-\pi, \pi)$ with $\theta_{n}$ $\rightarrow 0$. Let $\left\{I_{n}\right\}_{1 \leq n<\infty}$ be a sequence of disjoint open intervals in $(-\pi, \pi)$ with $\theta_{n} \in I_{n}$. Let $\left\{\alpha_{n}\right\}_{1 \leqq n<\infty}$ be a sequence of positive numbers such that $\sum_{1 \leqq n<\infty} \alpha_{n}<\infty$. The form of the Poisson kernel shows that if we take $r_{n}>0$ less than but close enough to 1 and set $Z_{n}=r_{n} e^{i \theta_{n}}$ then 


$$
P_{n}<\alpha_{n} \quad \text { on }[-\pi, \pi] \backslash I_{n} .
$$

Finally, select $\epsilon>0$ so that

$$
\sigma \equiv \epsilon\left(1+\sum_{1 \leqq n<\infty} \alpha_{n}\right)<1
$$

Let $\tilde{C}_{n}=\epsilon \gamma_{n}^{-1}, 1 \leqq n<\infty$. Since any $\theta \in[-\pi, \pi]$ is in at most one $I_{n}$ and since $\gamma_{n} \geqq 1$, (4) leads to

$$
\sum_{1 \leq n<\infty} \tilde{C}_{n} P_{n} \leqq \sigma .
$$

So in view of (5) and (3), we can define $\tilde{\mu} \in M_{\phi}$ by (2) with $C_{n}=\tilde{C}_{n}$.

Let $\mu \in M_{\phi}$ be given by the sequence $\left\{C_{n}\right\}_{1 \leqq n<\infty}$. By (3), $C_{n} \leqq \gamma_{n}^{-1}$ $=\epsilon^{-1} \tilde{C}_{n}$, so $\mu_{n} \leqq \epsilon^{-1} \tilde{\mu}_{n}, 1 \leqq n<\infty$. By (2) $\mu_{0} \leqq \lambda$ while by (2), (5), (6) $(1-\sigma) \lambda \leqq \tilde{\mu}_{0}$; thus $\mu_{0} \leqq(1-\sigma)^{-1} \tilde{\mu}_{0}$. If we set $K=\max \left\{\epsilon^{-1},(1-\sigma)^{-1}\right\}$, we see that $\mu \leqq K \tilde{\mu}$. By $\left[1\right.$, IV. 5.1] $\tilde{\mu}$ is a core measure for $M_{\phi}$.

REMARKs. Examination of our estimates shows that if we preassign a constant $K>2$, we can arrange that $\mu \leqq K \tilde{\mu}$ for all $\mu \in M_{\phi}$. So if $0<\tau<1 / 2$ is preassigned, we may arrange that $\tilde{\mu}-\tau \nu \in M_{\phi}$ whenever $\tilde{\mu}+\nu \in M_{\phi}$.

It is also worth noting that every multiplicative positive measure for $\mathfrak{A}$ on $Y$ is an Arens-Singer measure (in fact, $\exp (\mathfrak{U})=\mathfrak{A}^{-1}$ ); on the other hand, $M_{\phi}$ contains but one Jensen measure (namely the case $C_{n}=0$, all $n$ ), and it is not a core measure.

\section{REFERENCES}

1. T. W. Gamelin, Uniform algebras, Prentice-Hall, Englewood Cliffs, N.J., 1969.

Yale University, New Haven, Connecticut 06520 